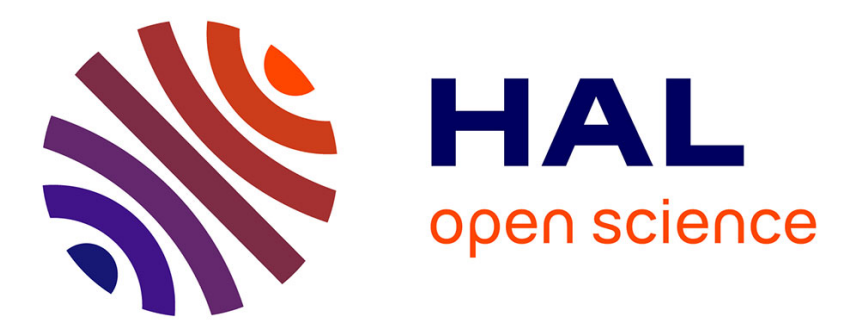

\title{
The microwave spectrum of the trans conformer of ethyl acetate
}

\author{
D. Jelisavac, D.C. C Cortés Gómez, Ha Vinh Lam Nguyen, L.W. W Sutikdja, \\ Wolfgang Stahl, I. Kleiner
}

\section{- To cite this version:}

D. Jelisavac, D.C. C Cortés Gómez, Ha Vinh Lam Nguyen, L.W. W Sutikdja, Wolfgang Stahl, et al.. The microwave spectrum of the trans conformer of ethyl acetate. Journal of Molecular Spectroscopy, 2009, 257 (2), pp.111-115. 10.1016/j.jms.2009.07.002 . hal-03183174

\section{HAL Id: hal-03183174 \\ https://hal.science/hal-03183174}

Submitted on 27 Mar 2021

HAL is a multi-disciplinary open access archive for the deposit and dissemination of scientific research documents, whether they are published or not. The documents may come from teaching and research institutions in France or abroad, or from public or private research centers.
L'archive ouverte pluridisciplinaire HAL, est destinée au dépôt et à la diffusion de documents scientifiques de niveau recherche, publiés ou non, émanant des établissements d'enseignement et de recherche français ou étrangers, des laboratoires publics ou privés. 


\title{
The Microwave Spectrum of the trans Conformer of Ethyl Acetate
}

\section{Jelisavac ${ }^{a}$, D.C. Cortés Gómez ${ }^{a}$, H.V.L. Nguyen ${ }^{a}$, L.W. Sutikdja ${ }^{a}$, W. Stahla ${ }^{\text {, and }}$}

\section{Kleiner}

a Institute of Physical Chemistry, RWTH Aachen University, Landoltweg 2, D-52074 Aachen, Germany

${ }^{\mathrm{b}}$ Laboratoire Interuniversitaire des Systèmes Atmosphériques (LISA), UMR 7583 (CNRS/Univ. Paris 12 \& Paris 7), Université de Paris 12, 61 avenue du Général de Gaulle, F-94010 Créteil cedex, France

Corresponding author: Wolfgang Stahl

Institute of Physical Chemistry
RWTH Aachen University
Landoltweg 2
D-52074 Aachen
Germany
Phone: +49 2418094724
Fax: +49 2418092365
Email: w.stahl@ rwth-aachen.de

\begin{abstract}
The Fourier transform microwave spectrum of ethyl acetate has been measured under molecular beam conditions. The trans conformer, where all heavy atoms are located within a mirror plane, was identified after analyzing the spectrum by comparison with theoretical calculations. The barrier to internal rotation of the acetate methyl group was found to be only $99.57(11) \mathrm{cm}^{-1}$ whereas for methyl torsion in the ethyl group a barrier of $1112.3(37) \mathrm{cm}^{-1}$ was determined. A comparison between two theoretical approaches treating the internal rotation, the so-called RAM (Rho-Axis-Method) and CAM (Combined Axis Method), is also performed.
\end{abstract}

Keywords: Microwave spectroscopy, Ethyl acetate, Internal rotation 


\section{Introduction}

Ethyl acetate is a widely used solvent and it is also abundant in many fruits contributing to their odors. From a chemical point of view it is a small aliphatic ester, obtained by condensation of ethanol and acetic acid using some acid as catalyst. Surprisingly, to our knowledge only one electron diffraction study [1] deals with the structure of this important molecule in the gas phase and no microwave studies have been reported.

Sugino et al. [1] suggested that ethyl acetate exists in two conformers, the trans conformer which has $\mathrm{C}_{\mathrm{s}}$ symmetry with all heavy atoms being located within the mirror plane, and a gauche conformer with $\mathrm{C}_{1}$ symmetry. Both conformers are shown in Fig. 1. Here, we will report on microwave studies on the trans conformer only.

\section{$<<$ Fig. 1 approximately here $>>$}

Ethyl acetate has two methyl groups that could show internal rotation. For the acetate methyl group, henceforward called methyl group I, we expected a low barrier to internal rotation on the order of $100 \mathrm{~cm}^{-1}$, for the ethyl methyl group, henceforward called methyl group II, the barrier was expected to be considerably higher, on the order of $1000 \mathrm{~cm}^{-1}$.

The motivation for this work was predominantly the interest in accurate internal rotation parameters of methyl group I. Another motivation was a comparison of two different computer programs, BELGI-C $\mathrm{C}_{\mathrm{s}}$ and XIAM, both of them treating internal rotation effects in rotational spectra using the rho axis method (RAM) [2] and the combined axis method (CAM) [3], respectively. 


\section{Experimental}

All spectra were recorded using two molecular beam Fourier transform microwave (MB-FTMW) spectrometers in the frequency ranges 4 to $26.5 \mathrm{GHz}[4,5]$ and 26.5 to $40 \mathrm{GHz}$ [6], respectively. Ethyl acetate was obtained from Sigma-Aldrich, Steinheim/Germany, and used without further purification. A gas mixture containing 1 to $3 \%$ ethyl acetate in helium at a total pressure of 80 to $120 \mathrm{kPa}$ was used throughout. Helium was chosen as carrier gas because the rotational cooling is not as effective as with neon or argon. This enabled us to observe also relatively high $\mathrm{J}$ values with satisfactory intensity.

\section{Spectral Analysis}

At the beginning of our measurements broadband scans in the frequency range from 10.0 to 11.9 GHz were carried out. In total 65 lines were found, many of them were quite strong. All of them were remeasured at high resolution and almost all lines were broadened, some lines were clearly split by up to some $100 \mathrm{kHz}$. A typical spectrum is shown in Fig. 2. The instrumental resolution and the measurement accuracy are given in the caption of Fig. 2.

<< Fig. 2 approximately here $>$

In ethyl acetate the rotational lines are split due to two large amplitude motions, the internal rotation of methyl group I and methyl group II. For methyl group I we assumed the barrier to internal rotation to be almost the same as Sheridan at al. [7] found for the acetate methyl group in methyl acetate, which is $99.559(83) \mathrm{cm}^{-1}$. This is a rather low barrier and we expected very large A-E splittings from a few $\mathrm{MHz}$ to a few $\mathrm{GHz}$, depending on the respective transition.

The internal rotation of methyl group II should be comparable to that in ethyl chloride, where a barrier of 3602(10) $\mathrm{cal} \mathrm{mol}^{-1}$, which is $1260(4) \mathrm{cm}^{-1}$, was found [8]. This would cause only broadened lines or narrow splittings for those transitions observable in the molecular beam and it explains the broadened and split lines we observed. 
At first we tried to assign the A species (referred to methyl group I) spectrum treating it as an effective rigid rotor spectrum. Therefore we used rotational constants obtained from quantum chemical calculations on various levels of theory for the trans and the gauche conformer (Tab. 1). By trial and error some $a$-type R-branch transitions of the trans conformer could be identified yielding the $\mathrm{B}$ and $\mathrm{C}$ rotational constants. Later, also some $b$-type Q-branch transitions were assigned and also the A constant was fixed. This enabled us to predict the whole rigid rotor spectrum with sufficient accuracy to find all remaining A species lines and, subsequently, to fit the (effective) quartic centrifugal distortion constants. The standard deviation after fitting $60 \mathrm{~A}$ species transitions was $3 \mathrm{kHz}$, which is almost experimental accuracy. It should be noted that despite an intense search no $c$-type transitions were found, which means that the $c$ dipole moment component is near zero and which confirms that we indeed observed the trans conformer with a mirror plane perpendicular to the $c$ axis.

In a next step we predicted both, the A and E species (referred to methyl group I) transitions using program XIAM [3]. The barrier was taken from methyl acetate, approximately $100 \mathrm{~cm}^{-1}$, the angle between the internal rotor axis and the inertial $a$ axis we calculated from our $a b$ initio result on MP2/6-311++G** level to be approximately $45^{\circ}$. The inertia of the methyl group was fixed to $3.2 \mathrm{u} \AA^{2}$, which we considered to be a reasonable value found in many molecules where methyl internal rotation has been analyzed. The predicted spectrum was in close agreement with lines we observed in our scan and the assignment was straight forward for the $a$-type $\mathrm{R}$ branch transitions, where the A-E splittings were only on the order of 10 to $100 \mathrm{MHz}$. The assignment of $b$-type Q branch lines, split by a few $100 \mathrm{MHz}$ up to a few $\mathrm{GHz}$, was more difficult. Here the search for lines which form closed cycles in the energy level diagram turned out to be very helpful. Finally, 60 A species lines and $66 \mathrm{E}$ species lines could be assigned. Levels up to $\mathrm{J}=19$ and $K_{a}=4$ were included in the fit (see also Tab. S1). The results are given in Tab. 2, a complete list of all fitted transitions is available as supplementary material (Tab. S1 and S2). It should be noted that the experimental data are obtained from the vibrational ground state. In contrast, the values from Gau03 are equilibrium data.

The internal rotation of methyl group II causes the A species lines of methyl group I to split into doublets $\left(\left|\sigma_{1}, \sigma_{2}\right\rangle=|0,0>| 0,, \pm 1>\right)$, the E species lines into triplets $(| \pm 1,0>| \pm 1,, \pm 1>$ $\mid \pm 1, \mp 1>$ ). Here the torsional states are labeled by the torsional symmetries $\sigma_{1}$ and $\sigma_{2}$ of group I 
and II [9]. Sample calculations with XIAM have shown that the splittings of the A species lines are usually too narrow to be resolved. However the E species lines of some selected transitions were split by up to $80 \mathrm{kHz}$. With the splittings observed for three E species transitions (Table S3) and keeping all other parameters fixed we found the barrier of methyl group II to be $\mathrm{V}_{3}=1112.3(37) \mathrm{cm}^{-1}$. A typical splitting is shown in Fig. 3.

$<<$ Fig. 3 approximately here $>>$

Here the angle between the internal rotor axis and the principal $a$ axis $<(\mathrm{i}, \mathrm{a})=147.7^{\circ}$ was taken from ab initio calculations. 


\section{Theoretical calculations}

In order to get rotational constants and also the angle between the internal rotor axis and the $a$ axis as starting values for assigning the spectra, theoretical calculations were carried out at the workstation cluster of the Center for Computing and Communication at the RWTH Aachen University using the program package Gaussian 03 [10]. In all cases a fully optimized structure was obtained. Also the dipole moment components were calculated to get an impression of the relative strength of $a-, b$-, and $c$-type transitions. At first we focussed our calculations on the trans conformer to compare the results of DFT and MP2 calculations with various basis sets. From former DFT studies given by Nagy et al. [11] two stable conformers of ethyl acetate were known. Our calculations with different start geometries and full relaxation of all structural parameters yielded three conformers. The results are summarized in Tab. 1. We found that the 6-311++G** basis set used for the MP2 or B3LYP method gave reasonable agreement with the experimental results, therefore we used only this basis set for our calculations on the gauche and cis conformer. The cis conformer, has an energy of about $3 \mathrm{~kJ} / \mathrm{mol}$ above the trans conformer and appeared unlikely to be visible under molecular beam conditions. However, the optimized structural parameters of all conformers are given in Table S4.

\section{Results and Discussion}

The microwave spectrum of ethyl acetate has been analyzed by means of two different programs, XIAM [3, 12], and BELGI-C $\mathrm{s}_{\mathrm{s}}[2,12]$. XIAM sets up the Hamiltonian in the principal axis system of the entire molecule. The internal rotation operator of each top is set up in its own rho axes system and after diagonalization, the resulting eigenvalues are transformed (rotated) into the principal axis system. Only centrifugal distortion constants, but no higher order coupling terms between internal rotation and overall rotation are implemented. A global fit of A and E species transitions is possible, however in cases with rather low barriers E species transitions are not satisfactorily predicted. The situation can be improved by fitting the A an E state separately, using two sets of rotational constants, but taking the same set of centrifugal distortion and internal rotation constants. This method significantly reduces the uncertainties in the fit, however 
the physical meaning becomes somewhat unclear. In our case the A species could be fitted with a standard deviation of $2.9 \mathrm{kHz}$, but for the E species $85.3 \mathrm{kHz}$ was obtained.

As an alternative, a global fit with BELGI-C $\mathrm{C}_{\mathrm{s}}$ was carried out. In this program the calculations are carried out in the rho axes system (also referred in the literature often as RAM for "rho axis method"), and all parameters obtained in the fit are referred to the rho axes system. The method based on the work of Kirtman [13], Lees and Baker [14] and Herbst et al. [15] takes its name from the choice of the axis system, the rho axis system, which is related to the principal axis system by a rotation chosen to eliminate the $-2 \mathrm{FP}_{\gamma} \rho_{\mathrm{x}} \mathrm{J}_{\mathrm{x}}$ and $-2 \mathrm{FP}_{\gamma} \rho_{\mathrm{y}} \mathrm{J}_{\mathrm{y}}$ coupling terms in the kinetic energy operator where $\mathrm{F}$ is the internal rotation constant, $\mathrm{P}_{\gamma}$ is the internal angular momentum, $\mathrm{J}_{\mathrm{x}}$ and $\mathrm{J}_{\mathrm{y}}$ are the usual $\mathrm{x}$ and $\mathrm{y}$ components of the global rotation angular momentum and $\rho$ is a vector that expresses the coupling between the angular momentum of the internal rotation $\mathbf{P}_{\gamma}$ and the global rotation $\mathbf{J}$. Unlike XIAM, BELGI-C $\mathrm{C}_{\mathrm{s}}$ which was used successfully to describe the spectra for internal rotors with very low internal rotation barriers $\left(\mathrm{V}_{3} \cong 25 \mathrm{~cm}^{-1}\right)$ such as acetamide [16], and also for peptide mimetics such as the ethylacetamidoacetate molecule [17] and the $\mathrm{N}$-acetyl alanine methyl ester [18] allows for fitting many higher order terms not only in the total angular momentum $\mathbf{J}$, but also in the angular momentum of the internal rotor $\mathbf{P}_{\gamma}$ and in cross-terms between them. BELGI- $\mathrm{C}_{\mathrm{s}}$ uses a two-step diagonalisation procedure in which the first step is the diagonalisation of the torsional Hamiltonian consisting of the one dimensional potential function $\mathrm{V}(\gamma)$ together with a torsion-rotation kinetic operator diagonal in $\mathrm{K}$, the rotational quantum number. A first set of torsional calculations, one for each $\mathrm{K}$ values, is carried out using a $21 \times 21$ torsional basis set $:\left|K \mathrm{v}_{\mathrm{t}} \sigma\right\rangle=\exp [\mathrm{i}(3 \mathrm{k}+\sigma) \gamma]$ where $\mathrm{v}_{\mathrm{t}}$ is the principal torsional quantum number and $\mathrm{k}$ is an integer running from -10 to +10 for BELGI-C $\mathrm{s}$. For XIAM this indices k runs from -15 to 15 . This basis is then reduced by discarding all but the nine lowest torsional eigenfunctions for each K. Finally the torsional eigenfunctions are multiplied by the symmetric top rotational function $\mid \mathrm{J}, \mathrm{K}, \mathrm{M}>$ to form a basis set which is then used to diagonalize, in the second step, the zeroth-order asymmetric rotor terms and higher order terms in the Hamiltonian. 
Using BELGI-C $\mathrm{C}_{\mathrm{s}}$, the same data set of $60 \mathrm{~A}$ species and $66 \mathrm{E}$ species transitions was fitted with 15 parameters to experimental accuracy with a standard deviation of $2.9 \mathrm{kHz}$ for the A state and $3.0 \mathrm{kHz}$ for the E state. The results are presented in Tab. 2 and Tab. 3.

The standard deviation is in the same order for the A species for both programs, XIAM and BELGI-C $\mathrm{s}_{\mathrm{s}}$, but it is much smaller for the E species in the fit of BELGI-C $\mathrm{s}_{\mathrm{s}}$. Therefore the predictive power of BELGI-C $\mathrm{C}_{\mathrm{s}}$ is much better than XIAM, however, for assignment purposes $\mathrm{XIAM}$ is in some aspects more convenient to use since it is somewhat faster than BELGI-C.

In order to compare the results from BELGI- $\mathrm{C}_{\mathrm{s}}$ referring to a rho axes system with the more usual constants given in a principal axis system, some transformations can be made. $A_{\text {RAM, }} B_{R A M}$, $\mathrm{C}_{\mathrm{RAM}}$, and $\mathrm{D}_{\mathrm{AB}}$ are proportional to the elements of the inverse inertia tensor. Diagonalizing it by rotation around the $c$ axis by an angle $\theta_{R A M}$ yields the PAM constants A and B:

$$
\begin{aligned}
& A=\frac{1}{2}\left(A_{R A M}+B_{R A M}+\sqrt{\left(A_{R A M}-B_{R A M}\right)^{2}+4 D_{a b}^{2}}\right) \\
& B=\frac{1}{2}\left(A_{R A M}+B_{R A M}-\sqrt{\left(A_{R A M}-B_{R A M}\right)^{2}+4 D_{a b}^{2}}\right)
\end{aligned}
$$

with

$$
\tan \left(2 \theta_{R A M}\right)=\frac{2 D_{a b}}{\left(A_{R A M}-B_{R A M}\right)} .
$$

For the trans-ethyl acetate molecule this is $13^{\circ}$.

The centrifugal distortion constant $\mathrm{D}_{\mathrm{J}}$ has the same meaning in both coordinate systems, because the $\mathrm{J}^{4}$ operator is invariant under rotation.

To determine the rotational constant $\mathrm{F}_{0}$ of the internal rotor, we start with the definition of the $\vec{\rho}$ vector $\vec{\rho}=\left(\rho_{a}, \rho_{b}, \rho_{c}\right)$. Its elements are defined by

$$
\rho_{a}=\frac{\lambda_{a} I_{\gamma}}{I_{a}}, \rho_{b}=\frac{\lambda_{b} I_{\gamma}}{I_{b}}, \rho_{c}=\frac{\lambda_{c} I_{\gamma}}{I_{c}},
$$

where $I_{a}, I_{b}, I_{c}$ are the principal moments of inertia of the entire molecule and $I_{\gamma}$ is the moment of inertia of the internal rotor. $\lambda_{\mathrm{a}}, \lambda_{\mathrm{b}}, \lambda_{\mathrm{c}}$ are the direction cosines between the internal rotor axis and the principal axes $a, b, c$, with 


$$
\lambda_{a}^{2}+\lambda_{b}^{2}+\lambda_{c}^{2}=1
$$

The relations (4) may also be expressed with the respective rotational constants $\mathrm{A}, \mathrm{B}, \mathrm{C}$, and $\mathrm{F}_{0}$ of the molecule and the internal rotor

$$
\rho_{a}=\frac{\lambda_{a} A}{F_{0}}, \rho_{b}=\frac{\lambda_{b} B}{F_{0}}, \rho_{c}=\frac{\lambda_{c} C}{F_{0}} .
$$

Note that in relation (6) above, the definition of $\mathrm{F}_{0}$ is different from that of the "reduced' $\mathrm{F}$ given in $\mathrm{Eq}(9)$ of [2] :

$$
F=\frac{\hbar^{2}}{2 r I_{\gamma}} \text { with } r=1-I_{\alpha}\left(\frac{\lambda_{a}^{2}}{I_{a}}+\frac{\lambda_{b}^{2}}{I_{b}}+\frac{\lambda_{c}^{2}}{I_{c}}\right)
$$

The length of the vector $\vec{\rho}$ is given by

$$
\rho=\sqrt{\rho_{a}^{2}+\rho_{b}^{2}+\rho_{c}^{2}} .
$$

In our case $\lambda_{c}=0$, and with (5), (6), and (7) we obtain

$$
\rho^{2} F_{0}^{2}=\lambda_{a}^{2}\left(A^{2}-B^{2}\right)+B^{2} .
$$

The $a$ component of $\vec{\rho}$ is available from

$$
\rho_{a}=\rho \cos \theta_{R A M},
$$

and with (6) we find

$$
\lambda_{a}=\frac{F_{0} \rho_{a}}{A}
$$

The system of equations (8) and (10) may be solved to yield

$$
F_{0}=\frac{B}{\rho \sqrt{1-\frac{A^{2}-B^{2}}{A^{2}} \cos ^{2} \theta_{R A M}}}
$$

and

$$
\lambda_{a}=\sqrt{\frac{\rho^{2} F_{0}^{2}-B^{2}}{A^{2}-B^{2}}} .
$$

From (11) and (12) the angle between the internal rotor axis and the inertial $a$ axis is obtained by

$$
\angle(i, a)=\arccos \lambda_{a} .
$$


All results, obtained with XIAM, BELGI-C $\mathrm{C}_{\mathrm{s}}$, and by theoretical calculations, referred to the principal axis system, are summarized in Tab. 2.

The torsional barrier determined for the acetate methyl rotor is $99.57(11) \mathrm{cm}^{-1}$ using BELGI-C and 101.606(23) $\mathrm{cm}^{-1}$ using XIAM. These differences are within a few percent. The discrepancies are, however, larger than the standard deviation of the parameters by one order of magnitude and are likely a result of systematic errors in the models. The differences between the methyl rotor angles are only about $0.025^{\circ}$ when comparing the two methods.

Also the agreement with the theoretical results allows to conclude, that we indeed observed the trans conformer of ethyl acetate. This is also supported by the absence of $c$-type transitions, which indicates that a mirror plane perpendicular to the $c$ axis is present. Finally we can also note that the fit achieved with BELGI-C $\mathrm{C}_{\mathrm{s}}$, which reproduced the experimental data within experimental accuracy, did not required any out-of-plan type terms of symmetry $A_{2}$.

It should be noted, that with both, XIAM and BELGI-C $\mathrm{C}_{\mathrm{s}}$, a strong correlation between $\mathrm{V}_{3}$ and $\mathrm{I}_{\gamma}$ is present which is due to the fact that only $\mathrm{v}_{\mathrm{t}}=0$ ground torsional state transitions are included in the analysis. However, both programs converged at almost the same $\mathrm{I}_{\gamma}$.

We can compare the internal rotation parameters with those of the acetate methyl group of methyl acetate [7]. Here, $\mathrm{V}_{3}$ and $\mathrm{I}_{\gamma}$ are $99.559(83) \mathrm{cm}^{-1}$ and $3.2085(26) \mathrm{u} \AA^{2}$, respectively. For ethyl acetate we found $99.57(11) \mathrm{cm}^{-1}$ and 3.16067(76) $\mathrm{u}^{2}$. This is almost the same and there seems to be no influence of the alkyl group in alkyl esters. If this also holds for bigger alkyl groups we will study in future work.

The barrier of methyl group II is $1112.3(37) \mathrm{cm}^{-1}$, which is quite close to the barrier of $1260(4) \mathrm{cm}^{-1}$ found in ethyl chloride.

Finally it should be mentioned that only about $30 \%$ of all measured lines could be assigned. The still unassigned lines might be due to the gauche or other conformers, to excited torsional states, and also due to other vibrational states. Also some lines probably arise from isotopomeres of 
strong lines of trans ethyl acetate. However, due to the complexity of the spectrum, none of these species could be presently assigned.

\section{Acknowledgement}

We thank the Center for Computing and Communication of the RWTH Aachen University for free computer time and the Land Nordrhein-Westfalen for funds. 


\section{References}

[1] M.Sugino, H. Takeuchi, T. Egawa, and S. Konaka, J. Mol. Struct. 245 (1991) 357.

[2] J.T. Hougen, I. Kleiner, and M. Godefroid, J. Mol. Spectrosc. 163 (1994) 559.

[3] H.Hartwig and H.Dreizler, Z. Naturforsch. 51a (1996) 923.

[4] U. Andresen, H. Dreizler, J.-U. Grabow, and W. Stahl, Rev. Sci. Instrum. 61 (1990) 3694.

[5] J.-U. Grabow, W. Stahl, and H. Dreizler, Rev. Sci. Instrum. 67 (1996) 4072.

[6] I. Merke, W. Stahl, and H. Dreizler, Z. Naturforsch. 49a (1994) 490.

[7] J. Sheridan, W. Bossert, and A. Bauder, J. Mol. Spectrosc. 80 (1980) 1.

[8] W. Stahl, H. Dreizler, and M. Hayashi, Z. Naturforsch. 38a (1983) 1010.

[9] N. Ohashi, J. T. Hougen, R. D. Suenram, F. J. Lovas, Y. Kawashima, M. Fujitake, and J. Pyka, J. Mol. Spectrosc. 227 (2004) 28-42.

[10] Gaussian 03, Revision D.02, M. J. Frisch, G. W. Trucks, H. B. Schlegel, G. E. Scuseria, M. A. Robb, J. R. Cheeseman, J. A. Montgomery, Jr., T. Vreven, K. N. Kudin, J. C. Burant, J. M. Millam, S. S. Iyengar, J. Tomasi, V. Barone, B. Mennucci, M. Cossi, G. Scalmani, N. Rega, G. A. Petersson, H. Nakatsuji, M. Hada, M. Ehara, K. Toyota, R. Fukuda, J. Hasegawa, M. Ishida, T. Nakajima, Y. Honda, O. Kitao, H. Nakai, M. Klene, X. Li, J. E. Knox, H. P. Hratchian, J. B. Cross, V. Bakken, C. Adamo, J. Jaramillo, R. Gomperts, R. E. Stratmann, O. Yazyev, A. J. Austin, R. Cammi, C. Pomelli, J. W. Ochterski, P. Y. Ayala, K. Morokuma, G. A. Voth, P. Salvador, J. J. Dannenberg, V. G. Zakrzewski, S. Dapprich, A. D. Daniels, M. C. Strain, O. Farkas, D. K. Malick, A. D. Rabuck, K. Raghavachari, J. B. Foresman, J. V. Ortiz, Q. Cui, A. G. Baboul, S. Clifford, J. Cioslowski, B. B. Stefanov, G. Liu, A. Liashenko, P. Piskorz, I. Komaromi, R. L. Martin, D. J. Fox, T. Keith, M. A. Al-Laham, C. Y. Peng, A. Nanayakkara, M. Challacombe, P. M. W. Gill, B. Johnson, W. Chen, M. W. Wong, C. Gonzalez, and J. A. Pople, Gaussian, Inc., Wallingford CT, 2004.

[11] P.I. Nagy, F.R.Tejada, J.G. Sarver, and W.S. Messer, Jr., J. Phys. Chem. A (2004) 1017310185.

[12] The XIAM program and the Belgi-Cs program are both available at the web-site of Prof. Kisiel http://www.ifpan.edu.pl/ kisiel/prospe.htm

[13] B. Kirtman, J. Chem. Phys. 37 (1962) 2516-2539. 
[14] R. M. Lees and J. G. Baker, J. Chem. Phys. 48 (1968) 5299-5318.

[15] E. Herbst, J. K. Messer, F. C. DeLucia, and P. Helminger, J. Mol. Spectrosc. 108 (1984) 42-57.

[16] V. Ilyushin, E. A. Alekseev, S. F. Dyubko, I. Kleiner, and J. T. Hougen, J. Mol. Spectrosc. 227 (2004) 115-139.

[17] R. J. Lavrich, A. R. Hight Walker, D. F. Plusquellic, I. Kleiner, R. D. Suenram, J.T. Hougen and G. T. Fraser, J. Chem. Phys. 119 (2003) 5487-5504.

[18] D. F. Plusquellic, I. Kleiner, J. Demaison, R. D. Suenram, R. J. Lavrich, F. J. Lovas, G. T. Fraser and V. V. Ilyushin, J. Chem. Phys. 125 (2006) 104312. 


\section{Tables}

Table 1: Rotational constants (in GHz) and dipole moments (in Debye) of ethyl acetate (trans and gauche conformer) obtained by DFT and ab initio methods using the Gaussian 03 package.

\begin{tabular}{|c|c|c|c|c|c|c|c|}
\hline Method / Basis set & A & B & C & $\mu_{\mathbf{a}}$ & $\mu_{\mathbf{b}}$ & $\mu_{\mathrm{c}}$ & $\mu$ total \\
\hline \multicolumn{8}{|c|}{ trans conformer } \\
\hline B3LYP / 6-31++G** & 8.3797 & 2.0686 & 1.7122 & 1.19 & 1.79 & 0.00 & 2.15 \\
\hline B3LYP / $6-311++G * *$ & 8.4184 & 2.0738 & 1.7172 & 1.17 & 1.74 & 0.00 & 2.09 \\
\hline B3LYP/CC-PVTZ & 8.4576 & 2.0827 & 1.7246 & 1.08 & 1.70 & 0.00 & 2.01 \\
\hline $\mathrm{MP} 2 / 6-311 \mathrm{G} * \star$ & 8.3958 & 2.1069 & 1.7390 & 0.90 & 1.85 & 0.00 & 2.05 \\
\hline $\mathrm{MP} 2 / 6-311++\mathrm{G} * *$ & 8.3907 & 2.0994 & 1.7339 & 0.99 & 1.93 & 0.00 & 2.17 \\
\hline MP2/CC-PVTZ & 8.4491 & 2.1134 & 1.7452 & 0.93 & 1.92 & 0.00 & 2.13 \\
\hline \multicolumn{8}{|c|}{ gauche conformer } \\
\hline B3LYP/ 6-311++G** & 7.3909 & 2.2752 & 2.0174 & 0.61 & 1.79 & 0.13 & 1.90 \\
\hline $\mathrm{MP} 2 / 6-311++\mathrm{G}^{\star} \star$ & 7.2396 & 2.3602 & 2.0830 & 0.34 & 1.92 & 0.31 & 1.98 \\
\hline \multicolumn{8}{|c|}{ cis conformer } \\
\hline B3LYP/ $6-311++G^{*} *$ & 7.9554 & 2.0946 & 1.7113 & 2.69 & 3.94 & 0.00 & 4.77 \\
\hline $\mathrm{MP} 2 / 6-311++\mathrm{G} * *$ & 7.9442 & 2.1184 & 1.7269 & 2.83 & 4.41 & 0.00 & 5.24 \\
\hline Experimentala & 8.4117 & 2.0948 & 1.7341 & & & $0.00^{b}$ & \\
\hline
\end{tabular}

${ }^{\text {a }}$ This work, rounded, for exact rotational constants see Tab. 2 .

${ }^{\mathrm{b}}$ Concluded from the absence of $c$-type lines. 
Table 2: Molecular constants of ethyl acetate (trans conformer) obtained with program XIAM and comparison with results of BELGI- $\mathrm{C}_{\mathrm{s}}$ and $a b$ initio results.

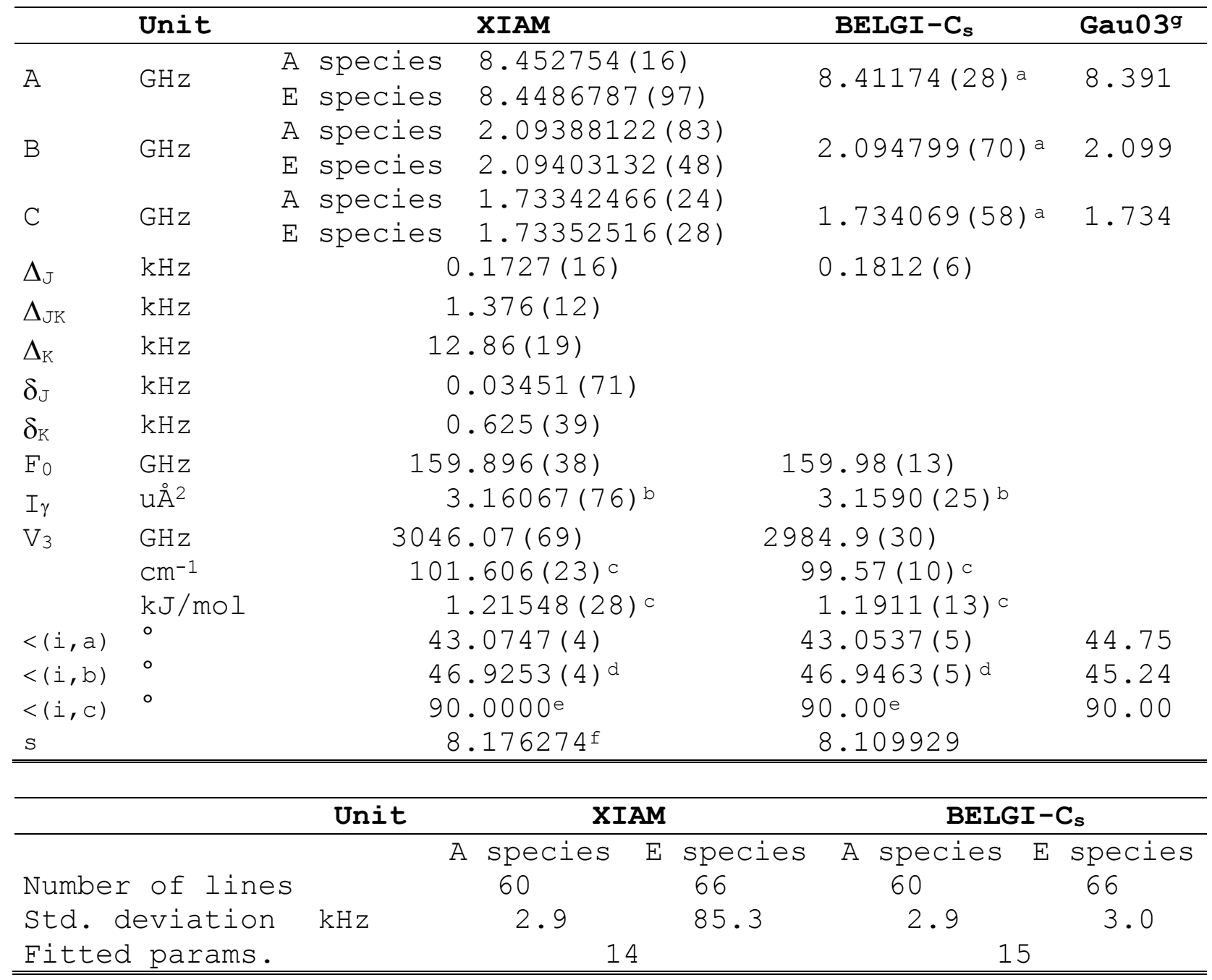

Note: All constants refer to the principal axis system, for the centrifugal distortion constants Watson's A reduction and a I r representations was used.

${ }^{a}$ Obtained by transformation from rho axis system to principal axis system, see text.

${ }^{\mathrm{b}}$ Moment of inertia $\mathrm{I}_{\gamma}$ of the internal rotor, calculated from its rotational constant $\mathrm{F}$.

${ }^{\mathrm{c}}$ Hindering potential, calculated from value in frequency units.

${ }^{\mathrm{d}}$ Calculated from $<(\mathrm{i}, \mathrm{b})=90^{\circ}-<(\mathrm{i}, \mathrm{a})$.

${ }^{\mathrm{e}}$ Fixed due to symmetry.

${ }^{\mathrm{f}}$ Reduced barrier $s=\frac{4 V_{3}}{9 F}$.

${ }^{\mathrm{g}}$ Calculation on MP2/6-311++G ${ }^{* *}$ level using Gaussian 03. 
Table 3: Molecular constants of ethyl acetate (trans conformer) obtained by a global fit using program BELGI-C .

\begin{tabular}{|c|c|c|c|}
\hline Operator & Constant ${ }^{b}$ & Unitc & Value \\
\hline $\mathrm{Pa}^{2}$ & A & $\mathrm{GHz}$ & $8.08735(41)$ \\
\hline $\mathrm{Pb}^{2}$ & B & $\mathrm{GHz}$ & $2.419190(32)$ \\
\hline $\mathrm{P}_{\mathrm{c}}{ }^{2}$ & $\mathrm{C}$ & $\mathrm{GHz}$ & $1.73406873(89)$ \\
\hline$\left\{\mathrm{P}_{\mathrm{a}}, \mathrm{P}_{\mathrm{b}}\right\}$ & $D_{A B}$ & $\mathrm{GHz}$ & $-1.394249(60)$ \\
\hline$-\mathrm{P}^{4}$ & $\mathrm{D}_{\text {J }}$ & $\mathrm{kHz}$ & $0.1812(5)$ \\
\hline$-\mathrm{P}^{2} \mathrm{P}_{\mathrm{a}}^{2}$ & DJK & $\mathrm{kHz}$ & $-0.349(17)$ \\
\hline$-2 \mathrm{P}^{2}\left(\mathrm{~Pb}^{2}-\mathrm{P}_{\mathrm{C}^{2}}\right)$ & $\delta_{\circlearrowleft}$ & $\mathrm{kHz}$ & $0.0372(1)$ \\
\hline $\mathrm{P} \gamma^{2}$ & F & $\mathrm{GHz}$ & $163.58(13)$ \\
\hline$(1 / 2)(1-\cos 3 \gamma)$ & $\mathrm{V}_{3}$ & $\mathrm{GHz}$ & $2984.9(30)$ \\
\hline $\mathrm{P}_{\mathrm{a}} \mathrm{P}_{\gamma}$ & $\rho$ & unitless & $0.039446(19)$ \\
\hline $\mathrm{P}_{\mathrm{a}}{ }^{3} \mathrm{P}_{\gamma}$ & $\mathrm{k}_{1}$ & $\mathrm{MHz}$ & $-0.2110(31)$ \\
\hline$(1-\cos 3 \gamma) \mathrm{P}_{\mathrm{a}}^{2}$ & $\mathrm{~K}_{5}$ & $\mathrm{MHz}$ & $114.6(12)$ \\
\hline$(1-\cos 3 \gamma) \mathrm{P}^{2}$ & $\mathrm{~F}_{\mathrm{V}}$ & $\mathrm{MHz}$ & $-3.531(45)$ \\
\hline$(1-\cos 3 \gamma)\left(P_{b}^{2}-P_{c}^{2}\right)$ & $\mathrm{C}_{2}$ & $\mathrm{MHz}$ & $-1.781(45)$ \\
\hline$(1-\cos 3 \gamma)\left\{\mathrm{P}_{a}, \mathrm{P}_{b}\right\}$ & $d_{A B}$ & $\mathrm{MHz}$ & $-6.69(17)$ \\
\hline
\end{tabular}

Unit

A species

E Species

Number of lines

Standard deviation

$\mathrm{kHz}$

60

66

2.9

3.0

Note:

a All constants refer to a rho-axis system, therefore the inertia tensor is not diagonal and the constants cannot be directly compared to those of a principal axis system. $\mathrm{P}_{a}, \mathrm{P}_{b}, \mathrm{P}_{c}$ are the components of the overall rotation angular momentum, $\mathrm{P}_{\gamma}$ is the angular momentum of the internal rotor rotating around the internal rotor axis by an angle $\gamma \cdot\{\mathrm{u}, \mathrm{v}\}$ is the anti commutator $u v+v u$.

$\mathrm{b}$ The product of the parameter and operator from a given row yields the term actually used in the vibration-rotation-torsion Hamiltonian, except for F, $\rho$, and A, which occur in the Hamiltonian in the form $\mathrm{F}\left(\mathrm{P}_{\gamma-} \rho \mathrm{P}_{\mathrm{a}}\right)^{2}+\mathrm{AP}_{\mathrm{a}}^{2}$.

${ }^{c}$ Values of the parameters from the present fit. Statistical uncertainties are shown as one standard uncertainty in the last digit. 


\section{Figure Caption}

Fig. 1: Trans and gauche conformer of ethyl acetate.

Fig. 2: A typical spectrum of trans ethyl acetate.

The experimental resolution was $0.8 \mathrm{kHz}$, the typical experimental line width $12 \mathrm{kHz}$ as indicated in the spectrum. The large splitting is due to the Doppler effect. For this spectrum 22 FIDs were co-added.

Fig. 3: The $303-2{ }_{11}$ E-state transition of trans ethyl acetate.

The splitting is due to the internal rotation of the methyl group II. Doppler splittings are indicated. 
Figure 1

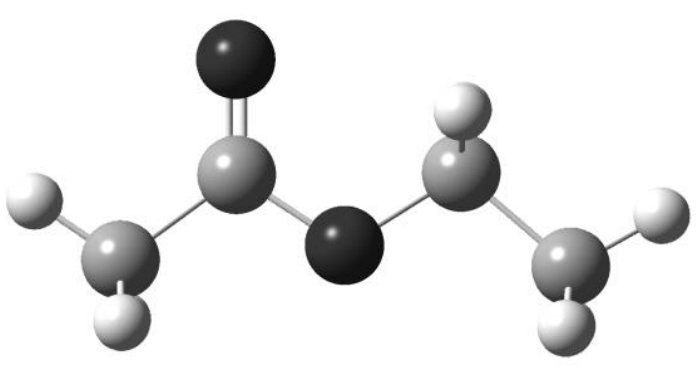

trans conformer

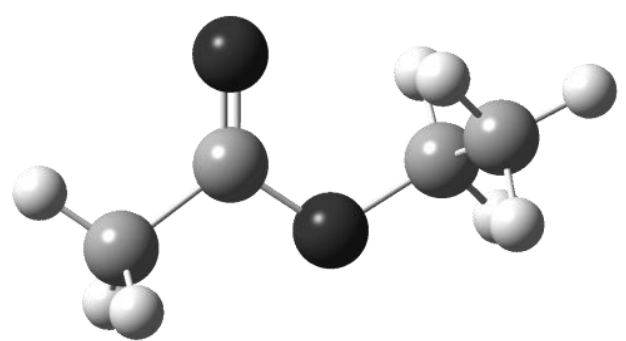

gauche conformer 
Figure 2

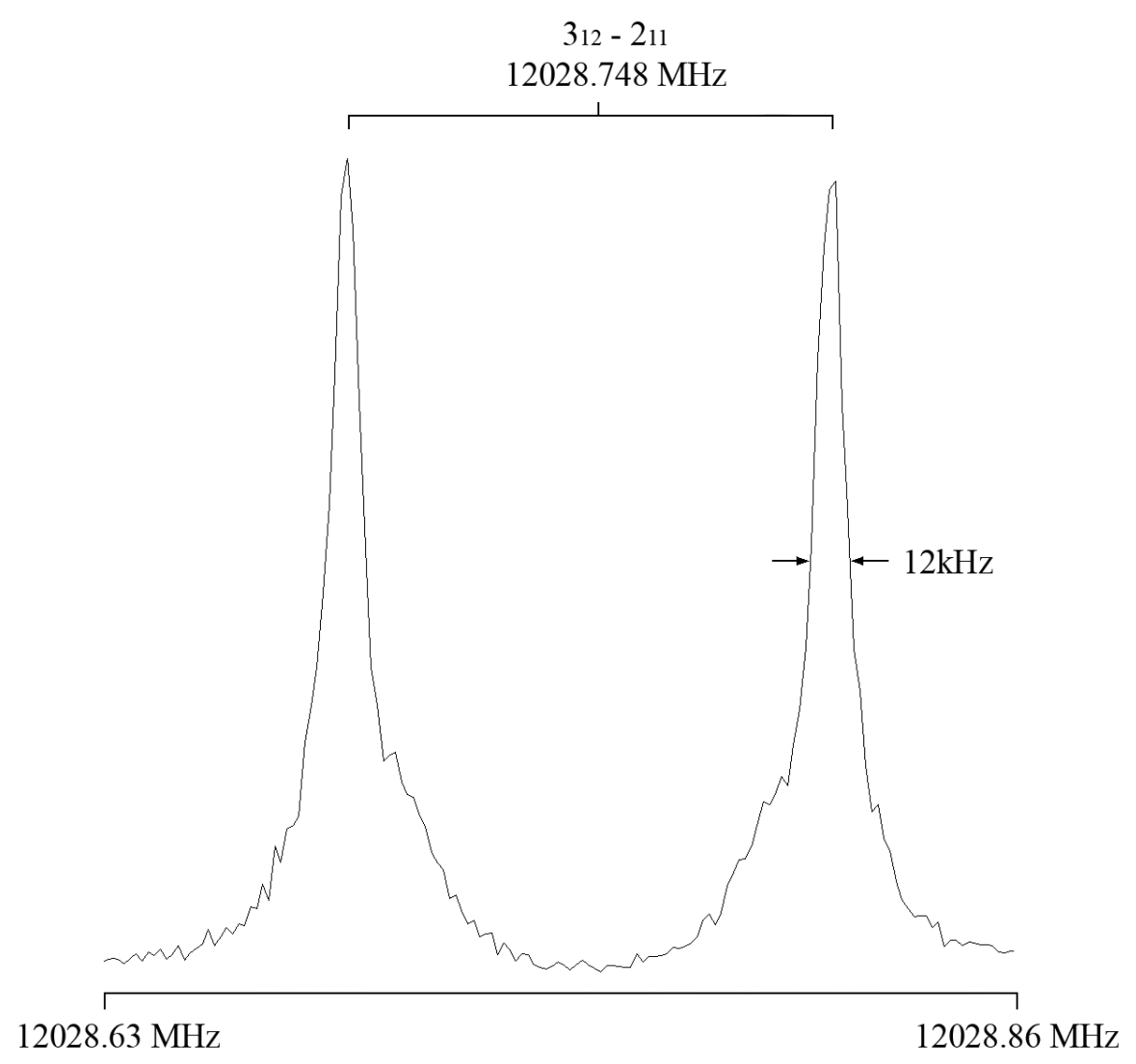


Figure 3

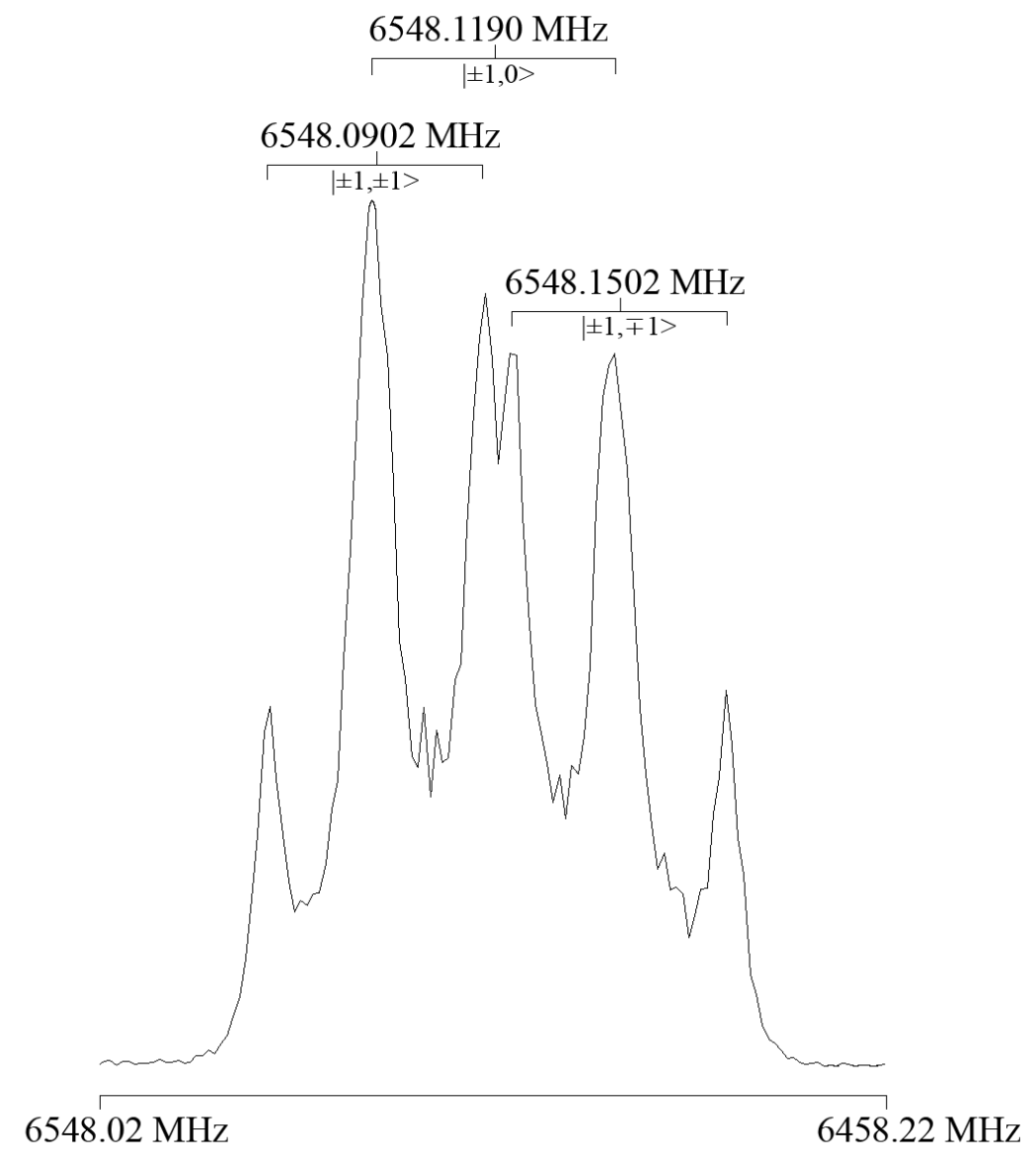

